\title{
Deterioration and maintenance models for insuring safety of civil infrastructures at lowest life-cycle cost ${ }^{1}$
}

\author{
Jan M. van Noortwijk ${ }^{2}$ and Dan M. Frangopol ${ }^{3}$
}

\begin{abstract}
The purpose of the paper is to describe and compare deterioration and maintenance models for civil infrastructures. These models can be applied to determine the best maintenance strategy to insure an adequate level of safety at minimal life-cycle cost while taking the uncertainties in the deterioration process into account. Without being complete, a time-dependent deterioration process can be modelled as a failure rate function, a Markov model, a stochastic process or a time-dependent reliability index. The pros and cons of the different models considered are discussed.
\end{abstract}

\section{Introduction}

This paper describes and compares deterioration and maintenance models for civil infrastructures that can be used to balance structural reliability and life-cycle cost. Civil infrastructure systems are designed for a particular service life and deteriorate with time. Maintaining these structures in a safe condition during their entire service life has been recognised as a very critical issue worldwide. According to Das (1999) there are two types of maintenance work: preventive maintenance which if it is not done it will cost more at a later stage to keep the structure in a safe condition, and essential maintenance which is required to keep the structure safe. Whilst it is easy to defend essential maintenance work on safety grounds, since failure consequences are in general extremely large, preventive treatments are more difficult to justify. The use of maintenance optimisation models is therefore of considerable interest for optimum life-cycle maintenance of deteriorating civil infrastructures.

An essential part of modelling maintenance is taking account of the uncertainties in the deterioration and the time of failure. In this paper, a brief overview is given on how to model uncertain deterioration for the purpose of maintenance optimisation. Without being complete, a time-dependent deterioration process can be modelled as (i) a failure rate function, (ii) a Markov model, (iii) a stochastic process or (iv) a time-dependent reliability index.

The outline of this paper is as follows. In the next four sections, the pros and

\footnotetext{
${ }^{1}$ Published in D.M. Frangopol, E. Brühwiler, M.H. Faber, and B. Adey, editors, Life-Cycle Performance of Deteriorating Structures: Assessment, Design and Management, pages 384-391. Reston, Virginia: American Society of Civil Engineers (ASCE), 2004.

${ }^{2}$ HKV Consultants, P.O. Box 2120, NL-8203 AC Lelystad, The Netherlands, and, Faculty of Electrical Engineering, Mathematics and Computer Science, Delft University of Technology, P.O. Box 5031, NL-2600 GA Delft, The Netherlands, E-mail: j.m.van.noortwijk@hkv.nl.

${ }_{3}^{3}$ Department of Civil, Environmental, and Architectural Engineering, University of Colorado, Boulder, CO 80309-0428, USA, E-mail: dan.frangopol@ colorado.edu.
} 
cons of the above-mentioned mathematical deterioration models are discussed by focussing on the maintenance decisions to be made. Next, the four deterioration models are compared. Finally, conclusions are formulated in the last section.

\section{Failure rate function}

A lifetime distribution represents the uncertainty in the time to failure of a component or structure. Let the lifetime have a cumulative probability distribution $F(t)$ with probability density function $f(t)$, then the failure rate function is defined as

$$
r(t)=\frac{f(t)}{1-F(t)}=\frac{f(t)}{\bar{F}(t)}
$$

(Barlow and Proschan, Chapter 2). A useful probabilistic interpretation of the failure rate function is that $r(t) d t$ represents the probability that a component of age $t$ will fail in the time interval $[t, t+d t]$. For deteriorating components or structures, the failure rate is increasing. Lifetime distributions and failure rate functions are especially useful in mechanical and electrical engineering. In these fields, one often considers equipment which can assume at most two states: the functioning state and the failed state. For example, a motor or switch is either working or not. A structure, on the other hand, can be in a range of states depending on its degrading condition. A serious disadvantage of failure rates is that they cannot be measured.

The area of optimising maintenance through mathematical models based on lifetime distributions was founded in the early sixties. This pioneering work is summarised in McCall (1965) and Barlow and Proschan (1965). Well-known models of this period are the age replacement model (replacement upon failure or upon reaching a predetermined age $k$, whichever occurs first) and the block replacement model (replacement upon failure and periodically at the times $k, 2 k, 3 k, \ldots$ ); see Barlow and Proschan (1965, Chapters 3-4). According to Dekker (1996) and Dekker and Scarf (1998), the age replacement model is one of the maintenance optimisation models that has been applied most.

\section{Markov model}

A Markov deterioration model is based on the assumption that the condition of a component can be described in terms of a limited number of condition states. Transition probabilities link the current state with a maintenance action to a future state. A transition probability is defined as the probability that a component will move from one state to another (same or worse one) depending on the action taken (including "no action"). The Markov property entails that the probability of deteriorating to another state doesn't depend on the history of the process, but only depends on the last condition and action. The time of transition from one state to another may have a probability distribution. Because a Markov deterioration model is condition-based, it is quite flexible in adapting it to (visual) inspection data and to incorporate inspections.

Examples of maintenance optimisation models based on Markovian deterioration are the Arizona Pavement Management System (Golabi et al., 1982; 
Wang and Zaniewski, 1996) and the Bridge Management System PONTIS (Golabi and Shepard, 1997; Thompson et al., 1998). These models are described below.

The Arizona Pavement Management System (APMS) uses linear programming to find the cost-optimal maintenance strategy. Roads are subdivided in lanes of fixed length (pavement segments) for which maintenance actions are formulated. Three deterioration characteristics are used to define the pavement condition: roughness, cracking, and index to the first crack (damage initiation time). They are discretised into 45 condition states (composed from three "roughness" states, three "cracking" states, and five "index to first crack" states).

The Bridge Management System PONTIS utilises the same Markovian linear programming approach as APMS. Bridge management, however, is more complex than road management. Bridges suffer from a lack of deterioration knowledge and data, don't allow for defining only a few uniform segments, have a great variety in type and design, and have many components deteriorating at different rates. For each component, PONTIS determines optimal maintenance actions for which the expected discounted cost over an unbounded time horizon is minimal.

As indicated in Frangopol and Das (1999) and Frangopol et al. (2001), the Markovian approach used in currently available bridge management systems has four important limitations, such as: (i) deterioration of a component is described in visual terms only; (ii) condition deterioration is assumed to be a single step function; (iii) future condition solely depends on the current condition (not on the deterioration history); and (iv) bridge system condition deterioration is not explicitly considered.

\section{Stochastic process}

A convenient way in modelling the uncertainty in time-dependent deterioration is by regarding it as a stochastic process. Examples of stochastic processes that can be used to model deterioration are the Brownian motion with drift (also called the Gaussian process) and the gamma process. A gamma process is a stochastic process with independent non-negative increments having a gamma distribution. A characteristic feature of the Brownian motion with drift is that a structure's resistance alternately increases and decreases. For this reason, the Brownian motion is inadequate in modelling deterioration which proceeds in one direction. In order for the stochastic deterioration process to proceed in one direction, it can best be considered as a gamma process. Because inspection measurements generally consist of cumulative amounts of deterioration, the advantage of the gamma process is evident. A related advantage of stochastic deterioration processes is that the modelling of inspections is quite natural and realistic. Even imperfect inspections can be dealt with, though the mathematical models become quite complex (Newby and Dagg, 2002; Kallen and Van Noortwijk, 2003).

Gamma processes have been applied to model the following deterioration processes: permanent coastal erosion of dunes (Van Noortwijk and Peerbolte, 2000), crest-level decline of dykes (Speijker et al., 2000), longshore rock transport near berm breakwaters (Van Noortwijk and Van Gelder, 1996), scour-hole development under the block mats of the Eastern-Scheldt barrier (Van Noortwijk and Klatter, 1999), current-induced rock displacement near the rock dumping of the Eastern-Scheldt 
barrier (Van Noortwijk et al., 1997), loss of steel thickness due to corrosion (Bakker et $a l ., 1999$ ), and corrosion of a hydrogen dryer (Kallen and Van Noortwijk, 2003).

On the basis of the above-mentioned gamma processes, tailor-made models have been built and implemented to determine the following cost-optimal maintenance decisions: optimal sand nourishment sizes and optimal dyke heightenings for which the expected costs of initial investment and future maintenance are minimal (Van Noortwijk and Peerbolte, 2000; Speijker et al., 2000); optimal inspection intervals for berm breakwaters, and for the block mats and rock dumping of the Eastern-Scheldt barrier for which the expected costs of inspection, maintenance and failure are minimal (Van Noortwijk and Van Gelder, 1996; Van Noortwijk and Klatter, 1999; Van Noortwijk et al., 1997); optimal intervals of lifetime extension by means of applying a protective steel coating for which the expected costs of lifetime extension and replacement are minimal (Bakker et al., 1999); and optimal inspection intervals for a hydrogen dryer for which the expected costs of inspection and maintenance are minimal (Kallen and Van Noortwijk, 2003). For those situations in which maintenance actions can be regarded as renewals bringing a component back to its original condition, renewal reward theory has been used to compute the expected costs (Rackwitz, 2001; Van Noortwijk, 2003).

As a basis for optimising maintenance, the Dutch Ministry of Transport, Public Works and Water Management (Rijkswaterstaat) implemented the age replacement model with discounted cost. This model has been applied for justification and optimisation of maintenance measures in the Netherlands (Klatter et al., 2002); detailed information on this model can be found in Van Noortwijk (1998) and Bakker et al. (1999). The criterion of expected discounted cost (net present value) over an unbounded horizon is used for comparing maintenance decisions. With the maintenance model, the expected discounted costs over a bounded horizon can also be calculated. In situations with a bounded time horizon larger than 50 years, the cost over an unbounded horizon may serve as a good approximation. By using this criterion, the cost of preventive maintenance can be balanced against the cost of corrective maintenance. For a discussion about which cost-based criterion can best be used, we refer to Van Noortwijk and Peerbolte (2000).

In Rijkswaterstaat's model, ageing has been modelled by a gamma deterioration process, where failure is defined as the event in which - due to deterioration - the condition at time $t$, denoted by the resistance $R(t)$, drops below the failure condition $s$. It is assumed that the expected deterioration at time $t$, denoted by $E(X(t))$, can be described using a power law; that is, the expected deterioration at time $t$ can be written as $a t^{b}$ for $a, b>0$. The optimal preventive replacement interval follows from an age replacement model based on a lifetime distribution. The probability of failure per year (discrete lifetime distribution) is defined as the probability that the condition drops below the failure condition per year.

This deterioration model has been extended for the possibility of lifetime-extending maintenance. With the extended model both the interval of lifetime extension and the interval of preventive replacement can be optimised. Through lifetime extension, the deterioration can be delayed so that failure is postponed and the lifetime of a component is extended (e.g., a coating protecting steel). Possible effects of lifetime-extending measures are the initiation period (time interval in which no 
deterioration occurs) and the condition improvement. Through replacement, the condition of a component is restored to its original condition.

\section{Time-dependent reliability index}

Experience gained in different countries shows that the major part of the work on existing bridges depends on the load carrying capacity (or structural reliability) of the bridge system rather than the condition states of the bridge elements alone (Frangopol and Das, 1999). Consequently, bridge management systems have to consider bridge reliability deterioration.

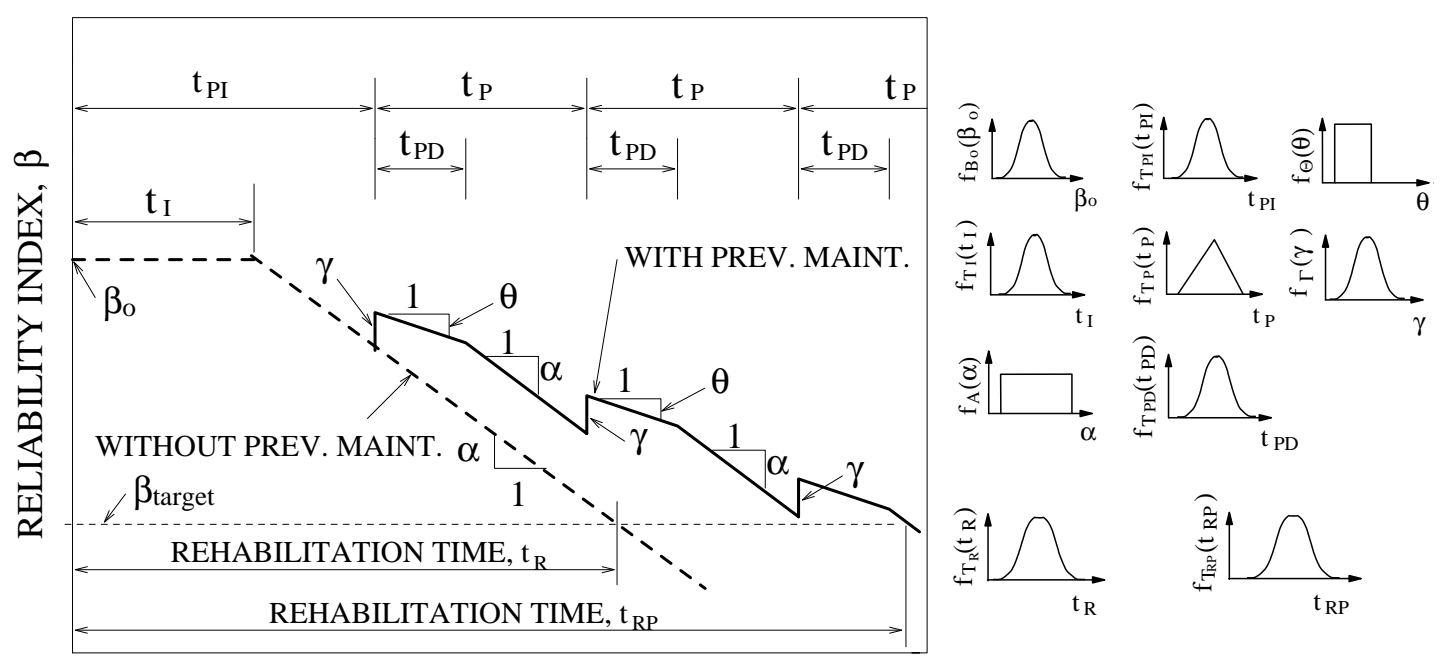

BRIDGE AGE. YEARS

Figure 1: Reliability Profiles and Associated Random Variables for the Options with or without Preventive Maintenance.

The reliability profile $\beta(t)$ is defined as the variation of the reliability index with time, (Thoft-Christensen, 1996; Estes and Frangopol, 1996; Nowak et al., 1998). Similar bridges designed and constructed to the same requirements, for various reasons, end up with different reliability levels (Frangopol and Das, 1999). This variation of reliability index influenced by various factors can be captured by using random variables. The eight random variables affecting the lifetime reliability profile of an individual deteriorating structure or a group of deteriorating structures under maintenance are shown in Figure 1 (Frangopol et al., 2001). These eight random variables are: (i) the initial reliability index $B_{0}$, (ii) the time of damage initiation $T_{I}$, (iii) the reliability index deterioration rate $A$ without lifetime extension, (iv) the time of first application of preventive lifetime-extending maintenance $T_{P I}$, (v) the time of reapplication of preventive lifetime-extending maintenance $T_{P}$, (vi) the duration of preventive lifetime-extending maintenance effect on reliability $T_{P D}$, (vii) the deterioration rate of the reliability index during preventive lifetime-extending maintenance effect $\Theta$, and (viii) the improvement in the reliability index (if any) immediately after the application of preventive lifetime-extending maintenance $\Gamma$. The probability density distributions of $B_{0}, T_{I}, A, T_{P I}, T_{P}, T_{P D}, \Theta$, and $\Gamma$, and their 
main descriptors for a group of steel/concrete composite bridges are indicated in Frangopol et al. (2001). Monte Carlo simulation is used to generate random samples from the probability density functions of the eight random variables $B_{0}, T_{I}, A, T_{P I}, T_{P}$, $T_{P D}, \Theta$, and $\Gamma$ and to capture the propagation of uncertainties during the entire service of existing deteriorating structures.

Reliability-based management represents the future generation of bridge management systems. An advantage of reliability-based maintenance/management is that the reliability is explicitly taken into account. A disadvantage is that the effects of maintenance (e.g., lifetime extension) on the reliability index are difficult to estimate.

\section{Model comparison}

In this section, the differences, similarities and interrelations between the four approaches to model deterioration are explained. Let us denote the initial resistance by $r_{0}$, the resistance (condition) at time $t$ by $R(t)$, the stress at time $t$ by $S(t)$, and the cumulative amount of deterioration by $X(t)$. Obviously, $R(t)=r_{0}-X(t)$. The interrelations between the four deterioration models are mathematically nicely summarised by the following formula:

$$
\beta(t)=-\Phi^{-1}(F(t))=-\Phi^{-1}\left(\operatorname{Pr}\left\{\min _{0 \leq u \leq t} R(u)-S(u)<0\right\}\right),
$$

where $\Phi$ is the normal cumulative distribution function. The failure rate function in Eq. (1), $r(t)$, is based on the lifetime distribution $F(t)$. Markovian deterioration focuses on $R(t)$, where the condition scale is discretised in terms of a limited number of condition states and the transition probabilities determine how deterioration proceeds in time. Stochastic deterioration processes model the uncertainty in the cumulative amount of deterioration $X(t)$. The time-dependent reliability index directly focuses on $\beta(t)$. Ideally, the best way is to base a deterioration model on the time-dependent stochastic processes of resistance and stress, and to compute the corresponding lifetime distribution and failure rate function, as well as the time-dependent reliability function. A disadvantage of a Markovian deterioration model is that the relation with the applied stress cannot be quantified accurately (because of the limited number of condition states).

\section{Conclusions}

Reliability-based deterioration and maintenance models represent the future generation of structure management systems. The advantage of reliability-based maintenance is that the reliability is explicitly taken into account. In condition-based deterioration models, the reliability only follows implicitly after transforming condition to reliability. The advantage of condition-based models is that conditions can be measured or inspected, whereas reliabilities must be computed, and that inspections can naturally be included in maintenance models. Ideally, the best way is to base a deterioration model on the time-dependent stochastic processes of resistance 
and stress, and to compute the corresponding lifetime distribution and failure rate function, as well as the time-dependent reliability function.

\section{Acknowledgements}

The work reported in this paper was supported by the Civil Engineering Division of the Dutch Ministry of Transport, Public Works, and Water Management, the U.S. National Science Foundation grants CMS-9912525 and CMS-0217290, and the U.K. Highways Agency. The authors thank Mr. Leo Klatter for helpful discussions. The opinions and conclusions presented in this paper are those of the authors and do not necessarily reflect the views of the sponsoring organizations.

\section{References}

Bakker, J.D., H.J. van der Graaf, and J.M. van Noortwijk (1999). Model of Lifetime-Extending Maintenance. In M.C. Forde, editor, Proceedings of the 8th International Conference on Structural Faults and Repair, London, United Kingdom, 1999. Edinburgh: Engineering Technics Press.

Barlow, R.E., and F. Proschan (1965). Mathematical Theory of Reliability. New York: John Wiley and Sons.

Das, P.C. (1999). Prioritization of bridge maintenance needs, Case Studies in Optimal Design and Maintenance Planning of Civil Infrastructure Systems, D.M. Frangopol, editor, ASCE, Reston, Virginia, 26-44.

Dekker, R. (1996). Applications of maintenance optimization models: a review and analysis. Reliability Engineering and System Safety, 51:229-240.

Dekker, R., and P.A. Scarf (1998). On the impact of optimisation models in maintenance decision making: the state of the art. Reliability Engineering and System Safety, 60:111-119.

Estes, A.C., and D.M. Frangopol (1996). Life-cycle reliability-based optimal repair planning for highway bridges: A case study. In D.M. Frangopol and G. Hearn, editors, Structural Reliability in Bridge Engineering, McGraw-Hill, New York, 54-59.

Frangopol, D.M., and P.C. Das (1999). Management of bridge stocks based on future reliability and maintenance costs. Bridge Design, Construction, and Maintenance, Institution of Civil Engineers, Thomas Telford, London, 45-58.

Frangopol, D.M., J.S. Kong, and E.S. Gharaibeh (2001). Reliability-based life-cycle management of highway bridges. Journal of Computing in Civil Engineering, ASCE, Vol. 15, No. 1, 27-34.

Golabi, K., R.B. Kulkarni, and G.B. Way (1982). A state-wise pavement management system. Interfaces, 12:5-21.

Golabi, K., and R. Shepard (1997). Pontis: A system for maintenance optimization and improvement of US bridge networks. Interfaces, 27:71-88.

Kallen, M.J., and J.M. van Noortwijk (2003). Inspection and maintenance decisions based on imperfect inspections. In Proceedings of the European Safety and Reliability Conference, Maastricht, The Netherlands, 2003.

Klatter, H.E., J.M. van Noortwijk, and N. Vrisou van Eck (2002). Bridge management 
in the Netherlands; Prioritisation based on network performance. In J.R. Casas, D.M. Frangopol, and A.S. Nowak, editors, First International Conference on Bridge Maintenance, Safety and Management (IABMAS), Barcelona, Spain, 14-17 July 2002. Barcelona: CIMNE.

McCall, J.J. (1965). Maintenance policies for stochastically failing equipment: a survey. Management Science, 11:493-524.

Newby, M., and R. Dagg (2002). Optimal inspection policies in the presence of covariates. In Proceedings of the European Safety and Reliability Conference, Lyon, France, 2002.

Nowak, A.S., Park, C.H., and Szersen, M.M. (1998). Lifetime reliability profiles for steel girder bridges. In D. M. Frangopol, editor, Optimal Performance of Civil Infrastructure Systems, ASCE, Reston, Virginia, 139-154.

Rackwitz, R. (2001). Optimizing systematically renewed structures. Reliability Engineering and System Safety, 73:269-279.

Speijker, L.J.P., J.M. van Noortwijk, M. Kok, and R.M. Cooke (2000). Optimal maintenance decisions for dikes. Probability in the Engineering and Informational Sciences, 14(1):101-121.

Thoft-Christensen (1996). Reliability profiles for concrete bridges. In D.M. Frangopol and G. Hearn, editors, Structural Reliability in Bridge Engineering, McGraw-Hill, New York, 239-244.

Thompson, P.D., E.P. Small, M. Johnson, and A.R. Marshall (1998). The Pontis bridge management system, Structural Engineering International, IABSE, 8(4):303-308.

Van Noortwijk, J.M. (1998). Optimal replacement decisions for structures under stochastic deterioration. In A.S. Nowak, editor, Proceedings of the Eighth IFIP WG 7.5 Working Conference on Reliability and Optimization of Structural Systems, Kraków, Poland, 1998, University of Michigan, Ann Arbor, 273-280.

Van Noortwijk, J.M. (2003). Explicit formulas for the variance of discounted life-cycle cost. Reliability Engineering \& System Safety, 80(2):185-195.

Van Noortwijk, J.M., M. Kok, and R.M. Cooke (1997). Optimal maintenance decisions for the sea-bed protection of the Eastern-Scheldt barrier. In R. Cooke, M. Mendel, and H. Vrijling, editors, Engineering Probabilistic Design and Maintenance for Flood Protection, Kluwer Academic Publishers, Dordrecht, 25-56.

Van Noortwijk, J.M., and E.B. Peerbolte (2000). Optimal sand nourishment decisions. Journal of Waterway, Port, Coastal, and Ocean Engineering, 126(1):30-38.

Van Noortwijk, J.M., and H.E. Klatter (1999). Optimal inspection decisions for the block mats of the Eastern-Scheldt barrier. Reliability Engineering and System Safety, 65(3):203-211.

Van Noortwijk, J.M., and P.H.A.J.M. Van Gelder (1996). Optimal maintenance decisions for berm breakwaters. Structural Safety, 18(4):293-309.

Wang, K.C.P., and J.P. Zaniewski (1996). 20/30 Hindsight: The new pavement optimization in the Arizona state highway network. Interfaces, 26:77-89. 